\title{
Empirical potentials for rovibrational energy transfer of hydrogen fluoride in collisions with argon
}

\author{
Robert M. Shroll ${ }^{\text {a) }}$ \\ Atmospheric, Oceanic and Space Sciences, University of Michigan, Ann Arbor, Michigan 48109-2143 \\ Lawrence L. Lohr \\ Department of Chemistry, University of Michigan, Ann Arbor, Michigan 48109-1055 \\ John R. Barker \\ Department of Chemistry and Department of Atmospheric, Oceanic and Space Sciences, University \\ of Michigan, Ann Arbor, Michigan 48109-2143
}

(Received 9 April 2001; accepted 7 June 2001)

\begin{abstract}
Rovibrational energy transfer of hydrogen fluoride in collisions with argon was investigated by using the coupled-states approximation to the quantum scattering problem. Empirically determined 3-D ab initio potential energy surfaces (PES) for the interaction between hydrogen fluoride and argon are presented. Second-order Møller-Plesset perturbation theory (MP2) was used to provide an initial approximate PES for the complex. The MP2 PES was subsequently modified to compensate for the underestimated dispersion interaction and adjusted until the desired agreement between calculated and observed spectroscopic quantities was achieved. Calculated rotational cross sections are in good agreement with experimental results as well as those obtained with a highly accurate vibrationally averaged empirical PES [J. M. Hutson, J. Chem. Phys. 96, 6752 (1992)]. The rate constants for the collision induced relaxation of the first vibrational state of hydrogen fluoride are presented as functions of temperature. The rate constants show structure at low temperature corresponding to cross-section resonances. The calculated rate constants are in good agreement with available high temperature experimental results. The calculations provide lower temperature rate constants and a wealth of detailed state-to-state information that are not available from experiment.

(c) 2001 American Institute of Physics. [DOI: 10.1063/1.1388547]
\end{abstract}

\section{INTRODUCTION}

The HF-Ar system is an important benchmark for the development and understanding of potential energy surfaces. There has been much experimental and theoretical interest in this system due mostly to the use of hydrogen fluoride in lasers. Even though there exists a great deal of experimental and computational data for the system, little is known about the details of its vibrational energy transfer. This may be attributed to both the experimental and the computational difficulties that are inherent to its study. One of the chief computational problems is the development of an accurate potential energy surface (PES). Here we test several methods for calculating the potential energy surface for the collisional vibrational energy transfer of this and similar systems. The surfaces generated are tested against known spectroscopic and rotational activation data. The final surfaces obtained are used to investigate rovibrational energy transfer of the $\mathrm{HF}-\mathrm{Ar}$ collisional system.

Vibrational energy transfer involving small molecules is of interest for a variety of reasons. In the atmosphere, highly vibrationally excited molecules (e.g., $\mathrm{CO}_{2}, \mathrm{O}_{3}, \mathrm{OH}, \mathrm{H}_{2} \mathrm{O}$, $\mathrm{NO}$ ) are produced as a result of chemical reactions, quenching of excited electronic states, and absorption of light. At

\footnotetext{
${ }^{a}$ Present address: Computational Biochemistry, Biophysics, and Biology Group, Environmental Molecular Sciences Laboratory, Pacific Northwest National Laboratory, Richland, WA 99352.
}

low altitudes, the high collision frequency maintains a Boltzmann vibrational energy distribution characterized by the local translational (kinetic) temperature: "Local thermodynamic equilibrium" (LTE). At high altitudes, "non-LTE" distributions are produced as the result of radiative and chemical processes in competition with collisions. Radiative energy is lost from the local atmosphere, affecting the efficiency of heat deposition. ${ }^{1-5}$ The occurrence of non-LTE can also compromise the interpretation of atmospheric limb radiances measured from satellites (e.g., Solomon et al. ${ }^{6}$ ). The present study was motivated by the desire to investigate nonLTE in $\operatorname{HF}(v)$, which is likely produced in atmospheric collisions of HF with $\mathrm{O}_{2}\left({ }^{1} \Delta\right)$ or $\mathrm{O}_{2}\left({ }^{1} \Sigma\right) .{ }^{7}$

The vibrational deactivation of $\mathrm{HF}$ in $\mathrm{HF}-\mathrm{Ar}$ mixtures has been studied in laser fluorescence and shock tube experiments, ${ }^{8-17}$ but accurate measurement of $\mathrm{HF}-\mathrm{Ar}$ energy transfer is difficult because it makes only a minor contribution compared to the much more efficient HF-HF energy transfer. At $295 \mathrm{~K}$ the rate of self-deactivation is reported as $\approx 2.0 \times 10^{-12} \mathrm{~cm}^{3} /$ molecule $\cdot \mathrm{sec}$, while the upper limit for the deactivation by argon at the same temperature is three orders of magnitude smaller. The experimental rate constants were determined from plots of inverse relaxation time versus HF mole fraction extrapolated to infinite dilution. At higher temperature, the $\mathrm{HF}-\mathrm{Ar}$ rate constants are measurable, but at low temperatures, the intercepts fall close to zero and experimental uncertainties prevent evaluation of the $\mathrm{HF}-\mathrm{Ar}$ rate 
constant. Thus, the results from the lower temperature experiments were reported as upper bounds. Computational prediction of the rate constant is also challenging since the dependence of the intermolecular potential on the HF vibrational coordinate must be assessed and the number of open rovibrational channels may be prohibitive. In contrast to the experiments, the computational procedure is simplified at lower temperatures because fewer channels are available.

There have been several previous theoretical studies of the collision induced deactivation of HF by $\mathrm{Ar}^{18-23}$ Ovchinnikova used a quasiclassical approximation with several simplifying transformations to calculate the vibrational deactivation rate constant from the first excited vibrational state with four different PESs. ${ }^{22}$ Two of them, which were based on previously determined self-consistent field (SCF) data, ${ }^{24}$ produced rate constants within the range of values obtained by experiments for temperatures between 800 and $4000 \mathrm{~K}$. Her results are not in close agreement with the present study, but they fall within the wide scatter of experimental values. Berend and Thommarson performed a quasiclassical trajectory study of HF $(v=1)$-Ar vibrational deactivation that provided good results for higher temperatures but overestimated the upper limits set by experimental observations at 294 and $350 \mathrm{~K} .^{20}$ Thompson conducted quasiclassical trajectory calculations using an additive pair potential for several vibrational states of hydrogen fluoride. ${ }^{18,19} \mathrm{He}$ provided a detailed preliminary survey of the state-to-state cross sections, but did not determine the relative translational energy dependence of the cross sections. This dependence is required in order to calculate rate constants and is also helpful in interpreting the qualitative behavior of the collisional system. When this paper was nearing completion, Krems et al. ${ }^{23}$ released a quantum mechanical study of the vibrational relaxation of the $\mathrm{HF}-\mathrm{Ar}$ system. Their intermolecular potential is based on the diatomics-in-molecule approach, ${ }^{25-27}$ which is much different than the augmented potentials used here. Their results are in good agreement with existing data.

The predicted rate constant for the vibrational deactivation of hydrogen fluoride by argon may be greatly improved by using better computational techniques and new spectroscopic data for the van der Waals complex. Recent advances in computer hardware have made it possible to use highly accurate quantum scattering methods such as the coupledstates (CS) approximation ${ }^{28}$ to calculate state-to-state cross sections. These same advances have made it possible to carry out more accurate and computationally demanding $a b$ initio calculations of the PES. Much is known now about the intermolecular potentials of van der Waals complexes and how to use $a b$ initio quantum mechanics to assess them reliably. ${ }^{29}$

There has been great interest in the development of intermolecular potential energy surfaces for prototype systems consisting of an atom and a diatomic molecule. ${ }^{24,30-50}$ Potential surfaces for the intermolecular interaction of hydrogen fluoride and argon have been reported numerous times. ${ }^{18-22,24-26,44-51}$ In Jacobi coordinates, the relative positions of the particles are represented by a vector $\mathbf{r}$ which points from the fluorine atom to the hydrogen atom, by a vector $\mathbf{R}$ which points from the diatomic center of mass to the argon atom, and by the angle $\theta$ between the vectors (the zero for $\theta$ is chosen to be the linear Ar-H-F geometry). In order to obtain a potential energy surface approaching spectroscopic accuracy directly from ab initio calculations, accurate electron correlation techniques must be employed with large basis sets and many different geometries. ${ }^{48,52}$ For most systems it is not yet practical to perform calculations with this level of accuracy or complexity and there is clearly a need for effective methods for enhancing the more modest $a b$ initio methods that are currently accessible.

Various approaches to potential energy surface enhancement have been reported. ${ }^{30-32,37,53-61}$ Some decompose the potential into physically meaningful terms that can be modified. . $^{31,37,54,55,61}$ Others scale the potential by a constant factor or scale the coordinates. ${ }^{56-60}$ Recently a technique was developed which scales both simultaneously. ${ }^{32}$ For an atomdiatom collisional system, spectroscopic data for van der Waals complexes may be used with nonlinear least squares algorithms to determine the modifications. ${ }^{45,62}$ In the present study we perform bound state calculations for the van der Waals complex and use the results to augment or morph a potential energy surface derived from second-order MøllerPlesset theory (MP2). We then determine the effects of the potential energy enhancements on the calculation of vibrational energy transfer.

Once a suitable potential is obtained, it is used to predict the rotational energy transfer cross sections and rate constants. These cross sections provide a test of the intermolecular potential that is a prelude to determining the vibrational rate constants. Rotational activation cross sections that have been measured ${ }^{63}$ at a relative translational energy of 350 $\mathrm{cm}^{-1}$ may be compared to highly accurate close-coupled theoretical calculations and are a useful test of the intermolecular potential energy surface anisotropy at the base of the repulsive wall. The CS calculations are used to predict rotational cross sections over the range of energies studied. Less is known experimentally about how the individual cross sections vary with the relative translational energy and therefore these dependencies cannot be compared directly to experimental data. However, the calculated energy dependence provides valuable insight into the qualitative behavior of the collisional system and is shown for selected cross sections. We also examine the relationship between the rotational cross sections corresponding to $\mathrm{HF}$ in different vibrational states and the suitability of the empirical power-gap law for data modeling and reduction. Representative rotational energy transfer rate constants are presented and discussed.

The vibrational energy transfer rate constants are calculated from the individual rovibrational state-to-state rate constants and are shown to be in good agreement with the limited experimental measurements. The original MP2 surface does well at calculating the vibrational deactivation rate constants. The surface enhancing techniques discussed here have little effect on the resulting vibrational energy transfer, however they do improve the predicted rotational activation cross sections and bound state energies. Analysis of the state-tostate rate constants shows a strong tendency toward pure V-R transitions. Qualitative structural features are evident for low-temperature rate constants, due to low energy crosssection resonance structures. All of the results are consistent 
with the available experimental observations and the known qualitative behavior of energy transfer cross sections and rate constants.

\section{POTENTIAL ENERGY SURFACES}

The empirical potentials developed here begin with a 3-D potential $V(R, \theta, r)$ based on a grid of points determined via MP2 calculations. The 3-D potential is modified either by scaling the MP2 potential or by adding terms that enhance the dispersion interaction, which is known to be underestimated by the MP2 level of electron correlation. This is a computationally simple step and could be replaced by one of the other methods of surface augmentation or morphing. Finding the best parameters is greatly accelerated by the existence of previously determined vibrationally averaged potentials (averaged over $r$ ), which may be used to make small improvements to the 3-D potential. By themselves these 2-D potentials have been used for rotational energy transfer, ${ }^{63}$ but they do not provide the necessary information to predict vibrational state changes.

The 3-D potential can be vibrationally averaged by integrating over the diatomic wave functions as shown in Sec. II C. The resulting 2-D potential is used to calculate the binding energy and spectroscopic properties of the van der Waals complex for comparison to experimental quantities. ${ }^{64-68}$ The MP2 PES is adjusted until satisfactory agreement is reached with the experimental spectroscopic properties. This method produces an improved 3-D potential energy surface for the van der Waals complex without a significant increase in the computational effort. By applying augmentation or morphing techniques to the 3-D surface and comparing the vibrationally averaged surface to experimental data, this technique preserves the dependence of the MP2 potential on the internal coordinate $r$ of the molecule.

Potential energy surfaces generated in the aforementioned manner are augmented based on the properties of their van der Waals potential wells. However, the collisional energy transfer cross sections and rate constants are mostly dependent on the repulsive part of the potential. The major deficiency of the MP2 surfaces is the underestimation of the attractive dispersion interaction ${ }^{29,69}$ which is most important near the van der Waals minimum. However, when the dispersion interaction is augmented, the repulsive wall of the potential is also modified. In order to determine the sensitivity of the energy transfer results to the form of the augmentation, three different augmented potentials are generated from an MP2 surface and used in scattering calculations.

\section{A. ab initio calculations}

The quantum chemistry method chosen for this study was second-order Møller-Plesset perturbation theory (MP2) with the aug-cc-pVTZ basis. ${ }^{70-72}$ This method has the advantage of being size consistent, which simplifies the determination of the intermolecular potential. Mourik and Dunning have recently performed $a b$ initio calculations for the HF-Ar system using this and other computational techniques. ${ }^{48}$ Their results show that the MP2 method is capable of reproducing important features of the potential energy surface, but the HF-Ar binding energy is underestimated even in the es- timated limit of a complete basis. For the HF-Ar complex, MP2 with the aug-cc-pVTZ basis recovers only approximately $80 \%$ of the binding energy $\mathrm{D}_{e}$. The deficiency is expected, since an accurate calculation of the attractive dispersion interaction requires highly correlated techniques. ${ }^{29,69}$ All of the MP2 calculations presented here were conducted using NWChem ${ }^{73}$ software.

The intermolecular potential $V(R, \theta, r)$ is obtained via the supermolecular approach ${ }^{29,38}$ and is expressed as

$$
\begin{aligned}
V(R, \theta, r)= & E_{\mathrm{HF}-\mathrm{Ar}}(R, \theta, r)-E_{\mathrm{HF}}(\infty, r)-E_{\mathrm{Ar}}(\infty) \\
& +\Delta E_{\mathrm{cp}}(R, \theta, r)-\Delta E_{\mathrm{sc}}(r),
\end{aligned}
$$

where $E_{\mathrm{HF}-\mathrm{Ar}}(R, \theta, r)$ is the potential energy of the whole system, $E_{\mathrm{HF}}(\infty, r)$ is the isolated diatom potential energy, $E_{\mathrm{Ar}}(\infty)$ is the isolated $\mathrm{Ar}$ atom potential energy, $\Delta E_{\mathrm{cp}}(R, \theta, r)$ is the counterpoise correction for the basis set superposition error (BSSE), and $\Delta E_{\mathrm{sc}}(r)$ is a size consistency correction.

The counterpoise correction is expressed as

$$
\begin{aligned}
\Delta E_{\mathrm{cp}}(R, \theta, r)= & E_{\mathrm{HF}}(\infty, r)-E_{\mathrm{HF}}(R, \theta, r) \\
& +E_{\mathrm{Ar}}(\infty)-E_{\mathrm{Ar}}(R, \theta, r),
\end{aligned}
$$

and substituting this result back into Eq. (1) gives

$$
V(R, \theta, r)=E_{\mathrm{HF}-\mathrm{Ar}}(R, \theta, r)-E_{\mathrm{HF}}(R, \theta, r)-E_{\mathrm{Ar}}(R, \theta, r),
$$

where $E_{\mathrm{HF}}(R, \theta, r)$ and $E_{\mathrm{Ar}}(R, \theta, r)$ are fragment energies requiring separate calculations. The size consistent term is equal to zero for the MP2 method. ${ }^{74}$ The dependence of $E_{\mathrm{HF}}(R, \theta, r)$ on $R$ and $\theta$ as well as the dependence of $E_{\mathrm{Ar}}(R, \theta, r)$ on $R, \theta$, and $r$ is due to the inclusion of the full set of basis functions that were used for the complex.

In order to obtain an accurate analytical representation of the potential $V(R, \theta, r)$, ab initio calculations were carried out for many values of $R, \theta$, and $r$. The range of $R$ fell between 1.5 and $20 \AA$ and included 23 unequally spaced points. This range was chosen to include values smaller than the lower integration limits of the subsequent scattering calculations (Sec. III A). The values of $r$ used were $0.7,0.9648$, $1.1,1.3$, and $1.5 \AA$. The value of $0.9648 \AA$ is the expectation value of $r$ for $v=1$ in isolated HF. The range of $r$ was determined from the HF vibrational wave-function amplitudes. The angle $\theta$ varied between $0^{\circ}$ and $180^{\circ}$ with 11 equally spaced values and thus covered the range of symmetry unique angles.

\section{B. Analytical representation of the interaction potential}

The $a b$ initio calculations provide a discrete set of points by which a functional representation must be determined. There are numerous ways of accomplishing this step, each with its own strengths. ${ }^{75-80}$ Here the potential function is represented by a Legendre polynomial expansion with coefficients determined using a matrix inversion technique. ${ }^{38,81}$ The potential may be expressed as 


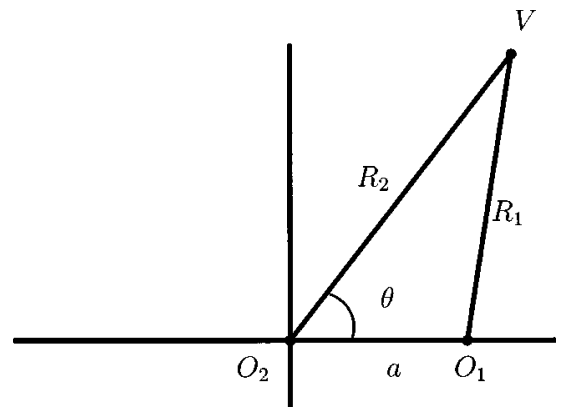

FIG. 1. Two coordinate systems for the expansion of the isotropic potential. The origin $\mathrm{O}_{1}$ corresponds to the center of the isotropic potential and the origin $\mathrm{O}_{2}$ corresponds to an off center expansion.

$$
\begin{aligned}
V(R, \theta, r) & =\sum_{k} \sum_{\lambda} V_{\lambda}^{k}(R) P_{\lambda}(\cos \theta)\left(r-r_{e}\right)^{k} \\
& =\mathbf{d}^{T} \mathbf{V}(R) \mathbf{s},
\end{aligned}
$$

where

$$
d_{\lambda}=P_{\lambda}(\cos \theta) \text { and } s_{k}=\left(r-r_{e}\right)^{k}
$$

and $P_{\lambda}(\cos \theta)$ are Legendre polynomials of order $\lambda$. The matrix $\mathbf{V}(R)$ is given by

$$
\mathbf{V}(R)=\mathbf{D}^{-1} \mathbf{B}(R) \mathbf{S}^{-1},
$$

where

$$
D_{\lambda m}=P_{\lambda}\left(\cos \theta_{m}\right) \text { and } S_{k n}=\left(r_{n}-r_{e}\right)^{k} .
$$

The $a b$ initio calculations are performed for the angles $\theta_{m}$ and the diatomic bond lengths $r_{n}$, constrained by the conditions $\lambda=m$ and $k=n$. These conditions are required by the matrix inversion step in Eq. (6). The terms $B_{\mathrm{mn}}(R)$ are represented by functions of the form

$$
\begin{aligned}
B_{\mathrm{mn}}(R)= & \exp \left[-a_{\mathrm{mn}}\left(R-R_{\mathrm{mn}}\right)\right]\left[\sum_{i=0}^{3} b_{\mathrm{mn}}^{(i)} R^{i}\right] \\
& -\tanh (R)\left[\sum_{i=3}^{5} c_{\mathrm{mn}}^{(2 i)} R^{-2 i}\right],
\end{aligned}
$$

where the coefficients $a_{\mathrm{mn}}, b_{\mathrm{mn}}^{(i)}$ and $c_{\mathrm{mn}}^{(2 i)}$ were determined using least squares. The hyperbolic tangent function in the last term was used as a damping function for the dispersion interaction. Various damping functions have been reviewed in the literature. ${ }^{30,69}$

The potential expansion convergence, with respect to the number of angular functions, is affected by the location of the coordinate origin. For example, an isotropic exponential repulsive potential may be expressed as,

$$
V\left(R_{1}\right)=A \exp \left(-\beta R_{1}\right),
$$

where $R_{1}$ is a distance from the first origin $O_{1}$ (see Fig. 1). Transforming to a second origin $\mathrm{O}_{2}$ gives,

$$
V\left(R_{2}, \theta\right)=A \exp \left\{-\beta R_{2}\left[\sum_{\lambda=0}^{\infty} P_{\lambda}(\cos \theta)\left(\frac{a}{R_{2}}\right)^{\lambda}\right]^{-1}\right\}
$$

with the constraint

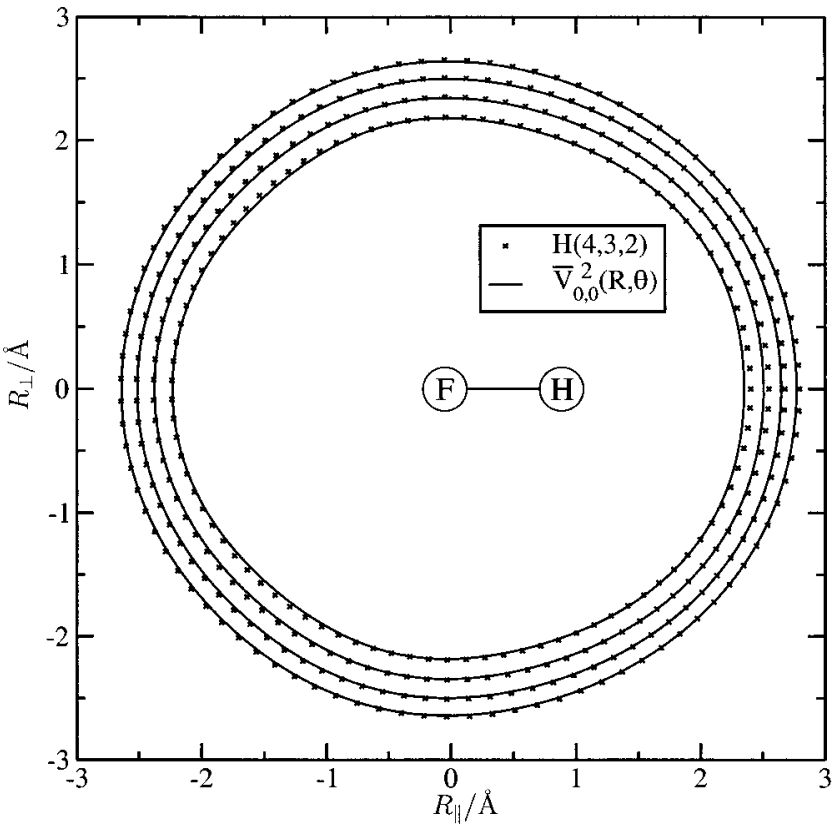

FIG. 2. The HF-Ar intermolecular potential classical turning points. The $\bar{V}_{0,0}^{2}(R, \theta)$ matrix element is shown with the $\mathrm{H} 6(4,3,2)$ potential for the ground vibrational state of HF. Each ellipse corresponds to a constant energy. From the outside ellipse inward, the energies are 1000, 2000, 4000, and $8000 \mathrm{~cm}^{-1}$. The HF-Ar center-of-mass is taken as the origin of the graph.

$$
R_{2}^{2}>\left|a^{2}-2 a R_{2} \cos \theta\right|,
$$

where $a$ is the magnitude of the displacement, $\theta$ is the angle between the position vector and the displacement vector as shown in Fig. 1 , and $a / R_{2}<1$. For $a=0$, only the $P_{0}$ term remains and the form of the original potential is obtained. For $a>0$, higher order Legendre polynomials are required. The expansion coefficients are proportional to $\left(a / R_{2}\right)^{\lambda}$ and it follows that for larger values of $a$ (greater displacement of the coordinate origin from the isotropic potential center) more terms are required for the expansion to converge. The same considerations apply to anisotropic potentials, however, the algebra is much more cumbersome.

The repulsive wall of the $\mathrm{HF}-\mathrm{Ar}$ potential closely resembles an ellipse (with nearly equal major and minor axes) as show in Fig. 2. The convergence of the angular part of Eq. (4) is expected to be best for a coordinate system with the origin located at the center of the ellipse. With the correct choice of origin for the HF-Ar potential, we also expect that the isotropic term should be the largest term for values of $R$ that correspond to the potential wall. Since Legendre polynomial expansions of the $\mathrm{HF}-\mathrm{Ar}$ intermolecular potential $V(R, \theta, r)$ are done with the origin located at the center-ofmass, we expect the convergence of the angular expansion to be best when the center of the ellipse is located close to the center-of-mass. Fortunately, this is the case for the MP2 potential.

An ellipse with the center located at $R_{\|}=0.06 \AA$ closely matches the classical turning points on the $V_{0,0}^{3}(R, \theta)$ potential at a relative translational energy of $1000 \mathrm{~cm}^{-1}$. The convergence of the expansion may be qualitatively seen by the relative magnitudes of the expansion coefficients $V(R)_{\lambda}^{k}$. For 


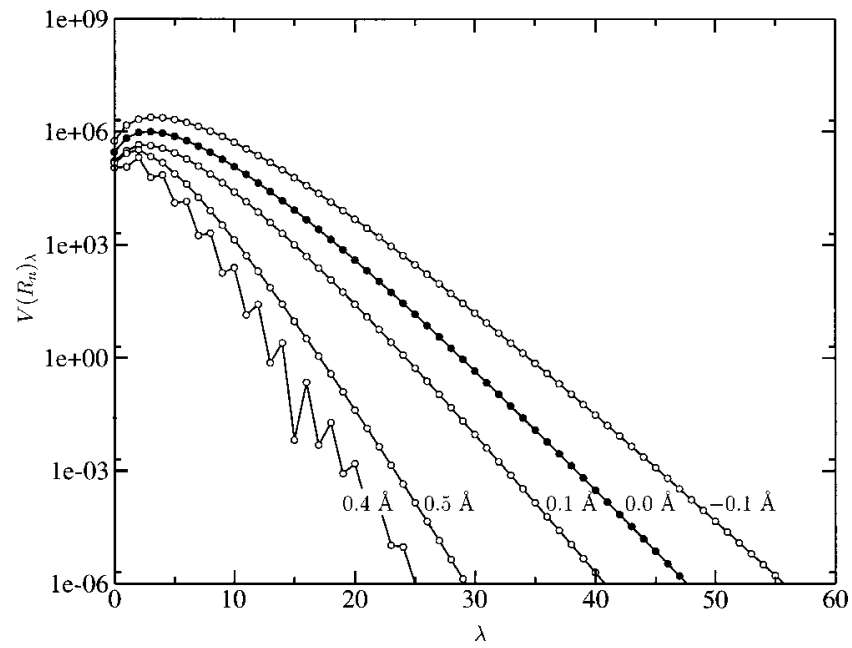

FIG. 3. The magnitude of the angular expansion coefficients for the Thompson (Refs. 18,19) potential. The radius is held constant $R_{n}=2.11 \AA$ for $n$ different polar coordinate origins. Numbers on the graph represent the displacement of the origin along the bond from the center-of-mass with a positive displacement toward the hydrogen. The coefficients for an origin at the center-of-mass have been emphasized. The plot for $0.4 \AA$ shows structure (smaller odd terms) that is due to the presence of symmetry.

$\mathrm{HF}-\mathrm{Ar}$ the isotropic term $V(R)_{0}^{0}$ is large and the Ar atom can only access regions in the scattering calculations where higher-order $(\lambda>4)$ Legendre polynomial expansions are small. For $R=2.12 \AA$, the $V(R)_{0}^{0}$ term is 8.4 times larger than the $V(R)_{4}^{0}$ term. Esposti et al. ${ }^{82}$ discuss this effect for similar systems, as well as provide several graphs of the expansion coefficients.

The same matrix technique was applied to Thompson's potential. Figure 3 shows the angular expansion coefficients for five different origin locations. From the graph it is evident that the best choice of origin is close to a $0.4 \AA$ displacement from the center of mass toward the hydrogen. A displacement of between 0.3 and $0.4 \AA$ is necessary to fit the repulsive wall to an ellipse at a classical turning point of $1000 \mathrm{~cm}^{-1}$. The saw tooth shape of the coefficients (smaller odd terms) indicates that for this choice of origin, the coordinates more correctly represent the symmetry of the potential. For a perfect ellipse, only even terms appear in the expansion. The variation in convergence between these sets of parameters is significant. For the matrix inversion technique, $\lambda$ can be interpreted as the number of angles in Eq. 7. For an $a b$ initio surface, optimizing the choice of origin could decrease the number of angles necessary to describe the potential.

\section{Surface augmentation}

Once an analytical representation was obtained, two new potential surfaces were generated by adjusting the expansion coefficients of Eq. (8). This allows the augmentation process to retain the same level of flexibility as the original fitting functions. In the present work the coefficients $c_{\mathrm{mn}}^{(2 i)}$ were modified until the potential reproduced observed binding energies $^{64-68}$ for the van der Waals complex to within the desired tolerance. The resulting surfaces differ in the form of Eq. (8) as shown by

$$
\begin{aligned}
& B_{\mathrm{mn}}^{1}(R)=B_{\mathrm{mn}}(R)-\tanh (R) \sum_{i=3}^{5} d_{m}^{(2 i)} R^{-2 i} \\
& B_{\mathrm{mn}}^{2}(R)=B_{\mathrm{mn}}(R)-\tanh (R) \sum_{i=3}^{5} d_{\mathrm{mn}}^{(2 i)} R^{-2 i},
\end{aligned}
$$

where the augmentation of $B_{\mathrm{mn}}^{1}(R)$ depends only on the angle $\theta$, and the augmentation of $B_{\mathrm{mn}}^{2}(R)$ depends on both the $r$ and $\theta$. The coefficients $d_{\mathrm{mn}}$ were assumed to vary linearly with $r_{n}$. Three-dimensional potentials are generated from Eq. (12) and Eq. (13) via Eq. (6).

The two augmented potentials were constructed in similar ways. The $V^{1}$ potential was augmented by adding an angular dependent dispersion interaction. The $V^{1}$ potential was made to reproduce the geometry and depth of the minima based on the highly accurate $a b$ initio calculations of Mourik and Dunning ${ }^{48}$ while reproducing the experimental spectroscopic bound state energies for the first five HF vibrational states of the van der Waals complex. ${ }^{64-68,83,84}$ The $V^{2}$ potential was augmented by adding a dispersion interaction that is a function of the angle $\theta$ and of the HF bond length. The H6 $(4,3,2)$ potential $^{45}$ was used to determine the angular dependence of the coefficients $d_{\mathrm{mn}}$ from Eq. (13). The $\bar{V}_{1,1}(R, \theta)$ element of the augmented potential was compared to the corresponding $\mathrm{HF}(v=1)$ surface of the $\mathrm{H} 6(4,3,2)$ potential. A least squares algorithm was used to minimize the difference between the potentials over all eleven angles and from $R=3.0 \AA$ to $R=5.0 \AA$. In this way information from the high quality vibrationally averaged potential $\mathrm{H} 6(4,3,2)$ was included during the construction of the 3 -D potentials. The angular dependence was determined from the H6(4, 3, 2) potential first, followed by the bond length dependence. The bond length dependence was assumed to be linear and determined by fitting the van der Waals complex bound state energies for the first three HF vibrational states. The stationary points of the potential energy surfaces are given in Table I.

\section{Potential scaling}

A third potential was obtain by scaling the MP2 potential by a constant factor

$$
V^{3}=1.23 V^{\mathrm{MP} 2}+V^{\mathrm{disp}}(\theta) .
$$

The coefficient was chosen to bring the van der Waals complex well depths into agreement with Mourik and Dunning. ${ }^{48}$ Spectroscopic binding energies for the van der Waals complex were then fitted by adding an angle-dependent dispersion interaction $V^{\text {disp }}(\theta)$ using Eq. (12). The angular dependence of this interaction was designed to broaden the wells. The stationary points of the potential energy surface are given in Table I.

\section{E. Bound state calculations}

In order to calculate the spectroscopic properties from the potentials and to perform quantum scattering calculations, a potential coupling matrix is required. The matrix was obtained from expectation values 
TABLE I. The potential energy surface stationary points for the total potential $V^{\text {tot }}=V_{\mathrm{HF}}^{\mathrm{RKB}}+V(R, \theta, r)$. The ab initio data were obtained from Mourik and Dunning. ${ }^{\text {a }}$

\begin{tabular}{lrrrr}
\hline \hline \multicolumn{5}{c}{ Minima } \\
Intermolecular potential & $\theta(\mathrm{deg})$ & $R(\AA)$ & $r(\AA)$ & $V\left(\mathrm{~cm}^{-1}\right)$ \\
\hline$V^{\mathrm{MP} 2}$ & 0.0 & 3.481 & 0.91761 & -171.95 \\
& 180.0 & 3.464 & 0.91701 & -74.49 \\
$V^{1}$ & 0.0 & 3.434 & 0.91760 & -207.04 \\
& 180.0 & 3.402 & 0.91703 & -93.42 \\
$V^{2}$ & 0.0 & 3.442 & 0.91764 & -184.09 \\
& 180.0 & 3.386 & 0.91712 & -103.11 \\
$V^{3}$ & 0.0 & 3.481 & 0.91779 & -211.58 \\
& 180.0 & 3.464 & 0.91705 & -91.63 \\
$\mathrm{CCSD}(\mathrm{T}) /$ aug-cc-pV5Z & 0.0 & 3.436 & 0.91811 & -206.38 \\
$\left.\mathrm{CCSD}^{\mathrm{a}} \mathrm{T}\right) /$ aug-cc-pVQZ & 180.0 & 3.415 & 0.91801 & -92.89 \\
& & & & \\
$V^{\mathrm{MP} 2}$ & Transition & states & & \\
$V^{1}$ & 93.72 & 3.638 & 0.91691 & -51.73 \\
$V^{2}$ & 101.56 & 3.484 & 0.91692 & -87.71 \\
$V^{3}$ & 124.99 & 3.453 & 0.91705 & -86.95 \\
$\mathrm{CCSD}(\mathrm{T}) /$ aug-cc-pVQZ & \\
\hline \hline
\end{tabular}

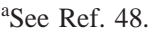

$$
\bar{V}_{v, v^{\prime}}(R, \theta)=\left\langle v, J=0|V(R, \theta, r)| v^{\prime}, J=0\right\rangle,
$$

where $|v, J=0\rangle$ are the diatomic vibrational wavefunctions. The vibrational wave-functions needed in Eq. (15) were determined from Numerov integration ${ }^{85,86}$ of RKR potentials ${ }^{87-89} V_{\mathrm{HF}}^{\mathrm{RKR}}$ using both software developed in house and the software program LEVEL. ${ }^{90}$

The program BOUND ${ }^{91,92}$ was used to perform closecoupling calculations of the bound state energies and to determine the binding energies $D_{0}$, the energy differences $E_{J=1}-E_{J=0}$, and the approximate centrifugal distortion constant $D_{J}=\left[-E_{J=2}+3 E_{J=1}-2 E_{J=0}\right] / 24$. Calculations were performed in the manner described by Chang et al., who performed bound state calculations on the H6(4, 3, 2) potential. ${ }^{93}$ Spectroscopic properties were calculated ignor- ing vibrational coupling, since the off-diagonal elements of the vibrational matrix do not greatly affect the eigenvalues. ${ }^{45}$

The spectroscopic properties calculated for all of the potentials are shown in Table II along with their experimental counterparts. The unmodified MP2 surface recovered $66 \%$ of the binding energy $D_{0}$, which is even less than the $80 \%$ recovery if measured from the bottom of the well $D_{e} \cdot{ }^{48}$ All of the augmented potentials reproduce the spectroscopic data reported in Table II significantly better than did the original MP2 surface.

\section{ROVIBRATIONAL ENERGY TRANSFER}

The HF-Ar collisional energy transfer may be represented by the following second-order reaction

$$
\mathrm{HF}\left(v_{i}, J_{i}\right)+\operatorname{Ar} \stackrel{k\left(v_{i}, J_{i}, v_{f}, J_{f} ; T\right)}{\longrightarrow} \mathrm{HF}\left(v_{f}, J_{f}\right)+\mathrm{Ar}
$$

where $v$ is the vibrational quantum number, $J$ is the rotational quantum number, $k\left(v_{i}, J_{i}, v_{f}, J_{f} ; T\right)$ is the temperature dependent rate constant, $i$ labels initial values, and $f$ labels final values. The rate constant is expressed in units $\mathrm{cm}^{3} /$ molecule. $\mathrm{sec}$ and is related to the reaction rate by

$$
\begin{aligned}
\frac{d\left[\operatorname{HF}\left(v_{f}, J_{f}\right)\right]}{d t} & =-\frac{d\left[\operatorname{HF}\left(v_{i}, J_{i}\right)\right]}{d t} \\
& =k\left(v_{i}, J_{i}, v_{f}, J_{f} ; T\right)\left[\operatorname{HF}\left(v_{i}, J_{i}\right)\right][\mathrm{Ar}],
\end{aligned}
$$

where square brackets denote number density in units of molecule $/ \mathrm{cm}^{3}$. The energy transfer rate constants are related to the state-to-state cross sections $\sigma\left(v_{i}, J_{i}, v_{f}, J_{f} ; E_{k}\right)$ using

\begin{tabular}{|c|c|c|c|c|c|c|c|}
\hline \multirow{2}{*}{\multicolumn{2}{|c|}{ Ground state (v000) }} & \multicolumn{3}{|c|}{$D_{0}\left(\mathrm{~cm}^{-1}\right)$} & \multicolumn{3}{|c|}{$E_{J=1}-E_{J=0}\left(\mathrm{~cm}^{-1}\right)$} \\
\hline & & $v=0$ & $v=1$ & $v=2$ & $v=0$ & $v=1$ & $v=2$ \\
\hline Expt. $^{b}$ & & 101.7 & 111.354 & 122.612 & 0.2045 & 0.20524 & 0.20597 \\
\hline$V^{1}$ & & 103.3335 & 111.2107 & 121.1713 & 0.203493 & 0.204372 & 0.205646 \\
\hline$V^{2}$ & & 101.7928 & 111.3201 & 122.6124 & 0.205927 & 0.207195 & 0.208654 \\
\hline$V^{3}$ & & 99.7093 & 109.6458 & 122.2411 & 0.196246 & 0.197723 & 0.199589 \\
\hline \multirow{3}{*}{\multicolumn{2}{|c|}{$V^{\mathrm{MP} 2}$}} & 67.1510 & 74.3351 & 83.4404 & 0.191739 & 0.193586 & 0.195814 \\
\hline & & \multicolumn{6}{|c|}{$D_{J}\left(\times 10^{-6} \mathrm{~cm}^{-1}\right)$} \\
\hline & & & $v=0$ & \multicolumn{2}{|c|}{$v=1$} & \multicolumn{2}{|l|}{$v=2$} \\
\hline \multicolumn{3}{|c|}{ Expt. $^{b}$} & 2.36 & \multicolumn{2}{|c|}{2.12} & \multicolumn{2}{|l|}{1.95} \\
\hline \multicolumn{3}{|c|}{$V^{1}$} & 2.50 & \multicolumn{2}{|c|}{2.25} & \multicolumn{2}{|l|}{2.17} \\
\hline \multicolumn{3}{|c|}{$V^{2}$} & 2.50 & \multicolumn{2}{|c|}{2.25} & \multicolumn{2}{|l|}{2.0} \\
\hline \multicolumn{3}{|c|}{$V^{3}$} & 2.25 & \multicolumn{2}{|c|}{2.04} & \multicolumn{2}{|l|}{1.88} \\
\hline \multicolumn{3}{|c|}{$V^{\mathrm{MP} 2}$} & 3.19 & \multicolumn{2}{|c|}{2.86} & \multicolumn{2}{|l|}{255} \\
\hline
\end{tabular}
the Maxwell-Boltzmann distribution

TABLE II. Spectroscopic properties of the potential energy surfaces. The binding energies $D_{0}$, state energy differences $E_{J=1}-E_{J=0}$, and a centrifugal distortion constant $D_{J}$ are presented. ${ }^{\text {a }}$

${ }^{a}$ For a description of the quantum numbers characterizing the states of the complex see Hutson (Ref. 45).

${ }^{\mathrm{b}}$ See Refs. 64-68. 


$$
\begin{aligned}
k\left(v_{i}, J_{i}, v_{f}, J_{f} ; T\right) & \\
= & 1 / k T\left(\frac{8}{\pi \mu k T}\right)^{1 / 2} \int_{0}^{\infty} E_{k} \sigma\left(v_{i}, J_{i}, v_{f}, J_{f} ; E_{k}\right) \\
& \quad \times \exp \left(-E_{k} / k T\right) d E_{k},
\end{aligned}
$$

where $E_{k}$ is the initial relative translational energy and $\mu$ is the $\mathrm{HF}-\mathrm{Ar}$ reduced mass. Rate constants for vibrational relaxation are obtained by averaging the state-to-state rate constants over the initial rotational states and summing over all final rotational states.

The experimental measurements cited herein have all been carried out on mixtures of $\mathrm{HF}$ and $\mathrm{Ar}$ in which the HF molecules undergo multiple collisions before being vibrationally deactivated. Since the cross sections for rotational energy transfer are much larger than those for vibrational deactivation, the rotational states are well-represented by a Boltzmann distribution. Therefore, the energy dependent cross section for vibrational energy transfer is given by

$$
\begin{aligned}
\sigma\left(v_{i}, v_{f} ; E_{k}\right)= & \frac{1}{q} \sum_{J_{i}}^{\text {open }}\left(2 J_{i}+1\right) \\
& \times \exp \left(-E_{J_{i}} / k T\right) \sigma\left(v_{i}, J_{i}, v_{f} ; E_{k}\right),
\end{aligned}
$$

where

$$
q=\sum_{J_{i}}\left(2 J_{i}+1\right) \exp \left(-E_{J_{i}} / k T\right),
$$

$E_{J_{i}}$ is the energy of the $J_{i}$ state, and

$$
\sigma\left(v_{i}, J_{i}, v_{f} ; E_{k}\right)=\sum_{J_{f}}^{\text {open }} \sigma\left(v_{i}, J_{i}, v_{f}, J_{f} ; E_{k}\right)
$$

is the cross section summed over all open final states. The thermal rate constant for vibrational energy transfer is given by

$$
\begin{aligned}
k\left(v_{i}, v_{f} ; T\right)= & \frac{1}{k T}\left(\frac{8}{\pi \mu k T}\right)^{1 / 2} \int_{0}^{\infty} E_{k} \sigma\left(v_{i}, v_{f} ; E_{k}\right) \\
& \times \exp \left(-E_{k} / k T\right) d E_{k} .
\end{aligned}
$$

\section{A. Quantum scattering calculations}

All quantum scattering calculations were performed using the HIBRIDON ${ }^{94-96}$ software package developed by M. H. Alexander and co-workers. For details, see the HIBRIDON distribution literature. Convergence of the calculated cross sections was verified with respect to all relevant parameters and was better than $2 \%$. A hybrid log-derivative/Airy propagator was used. ${ }^{97}$ Log-derivative integration was carried out from $4.0 \mathrm{a}_{0}$ to $30.0 \mathrm{a}_{0}$ with an interval of $0.05 \mathrm{a}_{0}$. Airy integration was used from $30.0 \mathrm{a}_{0}$ to $100.0 \mathrm{a}_{0}$ with a variable interval. A step size of 5 was used for the orbital angular momentum in the CS calculations. The reduced mass of the ${ }^{1} \mathrm{H}^{19} \mathrm{~F}$ and ${ }^{40} \mathrm{Ar}$ system is 13.331917 a.u. The vibrational coupling matrices were approximated as shown in Eq. (15). This approximation is expected to be least accurate for rate constants below room temperature; discussion of its validity may be found elsewhere. ${ }^{53,98,99}$
TABLE III. Relative cross sections for rotational activation of HF by $\mathrm{Ar}$ with a relative translational energy of $350 \mathrm{~cm}^{-1}$. Experimental values are given by Chapman et al. ${ }^{\text {a }}$ The results reported as $\overline{\mathrm{H}} 6(4,3,2)$ were determined from the H6 $(4,3,2)$ potential by Chapman et al. by using a $\pm 15 \%$ distribution of energies about the relative translational energy.

\begin{tabular}{cccccccc}
\hline \hline $\begin{array}{c}\text { Transition } \\
J_{f}-J_{i}\end{array}$ & Expt. & $\overline{\mathrm{H}} 6(4,3,2)$ & $\mathrm{H} 6(4,3,2)$ & $V^{1}$ & $V^{2}$ & $V^{3}$ & $V^{\mathrm{MP} 2}$ \\
\hline \multicolumn{7}{c}{ Percent of total cross section } \\
$1 \leftarrow 0$ & $77(8)$ & 76.9 & 77.18 & 81.20 & 77.70 & 89.49 & 94.51 \\
$2 \leftarrow 0$ & $18(3)$ & 18.3 & 18.36 & 13.62 & 18.36 & 7.60 & 3.58 \\
$3 \leftarrow 0$ & $4.4(8)$ & 4.44 & 4.45 & 5.18 & 3.94 & 2.91 & 1.91 \\
$4 \leftarrow 0$ & $0.4(4)$ & 0.280 & $\cdots$ & $\cdots$ & $\cdots$ & $\cdots$ & $\cdots$ \\
& \multicolumn{7}{c}{ Total cross section $\left(\AA^{2}\right)$} \\
& $25(5)$ & 21.3 & 21.10 & 16.89 & 20.24 & 15.53 & 17.53 \\
\hline \hline
\end{tabular}

${ }^{\text {a See Ref. } 63 .}$

For comparison with experimental results of Chapman et al., ${ }^{63}$ rotational cross sections were determined by solving the close-coupled quantum scattering equations for 350.0 $\mathrm{cm}^{-1}$ relative translation energy. The $\bar{V}_{0,0}(R, \theta)$ element of the potential coupling matrix was used from Eq. (15). The matrix element represents a vibrationally averaged potential that is appropriate for rotational energy transfer at low relative velocity.

The energy dependent rovibrational energy transfer cross sections, necessary for calculating the vibrational rate constants, were determined using the CS approximation. ${ }^{28,100-103}$ Using this approximation, the state-to-state cross sections are

$$
\sigma\left(v_{i}, J_{i}, v_{f}, J_{f} ; E_{k}\right)=\sum_{\Omega} \sigma^{\Omega}\left(v_{i}, J_{i}, v_{f}, J_{f} ; E_{k}\right),
$$

where $\Omega$ denotes the projection of the total angular momentum on the body-fixed quantization axis and the sum is over all values of the projection quantum number such that $|\Omega|$ $\leqslant \min \left(J_{i}, J_{f}\right)$. The CS approximation has been shown to be highly accurate for rovibrational energy transfer of atomdiatomic molecule collisions, except at very low relative velocities. For neutral systems, if the relative translational energy of both the initial and final states is greater than the well depth then the CS approximation is expected to be valid. ${ }^{104}$ Comparison between the CS approximation and closecoupling results shows no distinguishable differences for the vibrational deactivation cross section of $\mathrm{He}+\mathrm{H}_{2},{ }^{105,106}$ a system less anisotropic than $\mathrm{HF}-\mathrm{Ar}$. Kouri provides a detailed description of the CS approximation with its strengths and weaknesses. ${ }^{104}$ Rovibrational energy transfer cross sections were calculated over a range of total energies from 6117 to $16512 \mathrm{~cm}^{-1}$ relative to the bottom of the hydrogen fluoride intermolecular potential well.

\section{B. Rotational energy transfer}

The close-coupled rotational activation calculations are summarized in Table III for a relative translational energy of $350 \mathrm{~cm}^{-1}$. The experimental and theoretical results of Chapman et al., ${ }^{63}$ corresponded to a narrow distribution of relative translational energies centered at $350 \mathrm{~cm}^{-1}$. In their work, the width of the distribution opens the $J_{f}=4$ channel, which lies slightly above $350 \mathrm{~cm}^{-1}$. Their experimental re- 


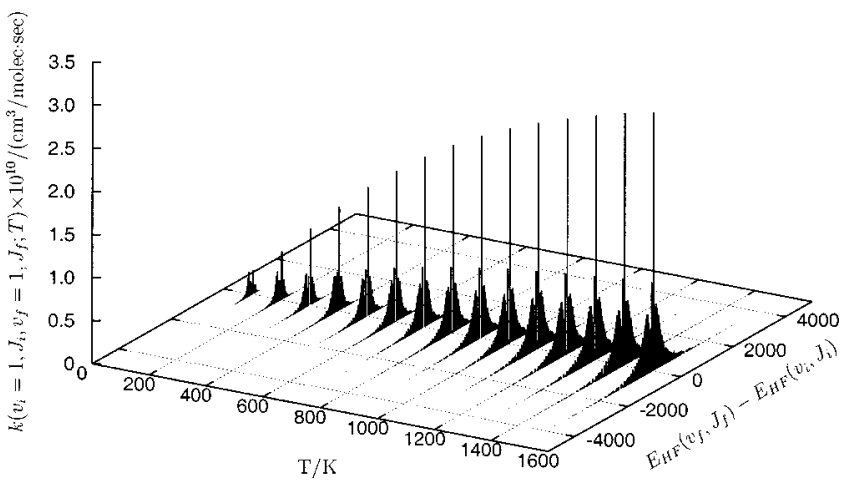

FIG. 4. The rotational rate constants $k\left(v_{i}=1, J_{i}, v_{f}=1, J_{f} ; T\right)$ for vibrationally elastic collisions of $\operatorname{HF}(v=1)$ and $\mathrm{Ar}$ as a function of temperature and the change in internal energy $E_{\mathrm{HF}}\left(v_{f}=1, J_{f}\right)-E_{\mathrm{HF}}\left(v_{f}=1, J_{i}\right)$ in wave numbers. The change in internal energy depends on the initial and final rotational angular momentum quantum numbers $J_{i}$ and $J_{f}$. Temperature is varied from 100 to $1500 \mathrm{~K}$ in increments of $100 \mathrm{~K}$. For each temperature, lines are plotted representing the rate constants for all open channels with $J_{f} \neq J_{i}$.

sults and their theoretical calculations based on Huston's H6 $(4,3,2)$ potential $^{45}$ are summarized in Table III by columns labeled Expt. and $\overline{\mathrm{H}} 6(4,3,2)$ respectively. All of the other data presented in Table III were calculated in the present study. A direct comparison with the experimental measurements may be made without taking into account the effect of the energy distribution, which is very narrow.

It is evident from the table that all of the potentials give qualitatively similar results. The results obtained with the $V^{2}$ potential agree with the experiments to within reported error. The $V^{2}$ potential is most accurate for the calculation of rotational cross sections and therefore it was used for all of the calculations presented for rotational energy transfer cross sections and rate constants. State-to-state rotational energy transfer rate constants were obtained using the CS approximation. An example of these rate constants as a function of temperature is shown in Fig. 4. The rate constants are for vibrationally elastic collisions of $\operatorname{Ar}$ with $\operatorname{HF}(v=1)$ where rotationally elastic terms have been omitted in order to show the relatively smaller inelastic terms more clearly. The figure shows a rapid decrease in the rate constant as a function of $\left|\Delta E_{\mathrm{HF}}\right|=\left|E_{\mathrm{HF}}\left(v_{f}, J_{f}\right)-E_{\mathrm{HF}}\left(v_{i}, J_{i}\right)\right|$. The exponential character of this decrease is evident for both upward and downward transitions shown in Fig. 5.

There has been much effort to create empirical models and fitting functions for rotational rate constants. ${ }^{107-124}$ Lang et al., observed rotational relaxation of $\mathrm{HF}$ in $\mathrm{HF}-\mathrm{Ar}$ mixtures and fitted their data to a simple exponential model. ${ }^{125}$ Their observations are consistent with ours: At a given temperature, higher $\mathrm{J}$ levels have lower probability of rotational deactivation, downward transitions are favored over upward ones for comparable $\left|\Delta E_{\mathrm{HF}}\right|$, and the rate constants decrease as $\left|\Delta E_{\mathrm{HF}}\right|$ increases.

The power-gap law has been used to model the matrix of $J_{i} \rightarrow J_{f}$ integral cross sections, ${ }^{111}$ but it is not accurate for the complete $J_{f}$-distribution. ${ }^{126}$ It has been shown that at least two sets of parameters are needed to fit the data: ${ }^{108}$ One set for $\left|\Delta E_{\mathrm{HF}}\right| \leqslant\left|\Delta E_{\mathrm{HF}}\right|^{*}$ and one set for $\left|\Delta E_{\mathrm{HF}}\right|>\left|\Delta E_{\mathrm{HF}}\right|^{*}$,

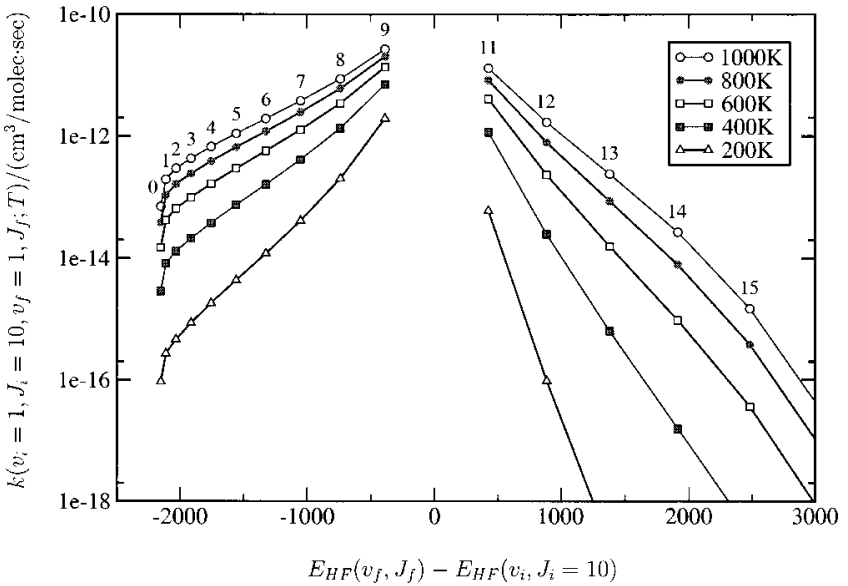

FIG. 5. The rotational rate constants $k\left(v_{i}=1, J_{i}=10, v_{f}=1, J_{f} ; T\right)$ for vibrationally elastic collisions of $\operatorname{HF}(v=1)$ and $\mathrm{Ar}$ as a function of temperature and the change in internal energy $E_{\mathrm{HF}}\left(v_{f}=1, J_{f}\right)-E_{\mathrm{HF}}\left(v_{i}=1, J_{i}=10\right)$ in wave numbers. Numbers located above data points represent the final rotational angular momentum quantum number for the series of temperatures. Data for the rotationally elastic transition $J_{i}=J_{f}=10$ have been excluded.

where $\left|\Delta E_{\mathrm{HF}}\right|$ is the energy gap between initial and final rotational levels and $\left|\Delta E_{\mathrm{HF}}\right| *$ is the intersection of the regressions (indicated by the vertical line in Fig. 6). The power-gap law for the cross sections can be written,

$$
\sigma\left(v_{i}, J_{i}, v_{f}, J_{f} ; E_{k}\right)=a\left(2 J_{f}+1\right)\left(E_{k}^{f} / E_{k}\right)^{1 / 2}\left|\Delta E_{\mathrm{HF}}\right|^{-\gamma},
$$

where $a$ and $\gamma$ are fitting parameters and $E_{k}^{f}$ is the kinetic energy of the final state. It follows from this equation that for one set of parameters $\{a, \gamma\}$ a plot of $\ln \left[\sigma^{\Omega}\left(v_{i}, J_{i}\right.\right.$ $\left.\left.=0, v_{f}, J_{f} ; E_{k}\right)\left(E_{k} / E_{k}^{f}\right)^{1 / 2} /\left(2 J_{f}+1\right)\right]$ versus $\ln \left|\Delta E_{\mathrm{HF}}\right|$ should yield a straight line. An example of such a plot is presented in Fig. 6, where two sets of parameters were determined by separate linear regressions over the regions to the left and right of the vertical line. The plot is for pure-rotational tran-

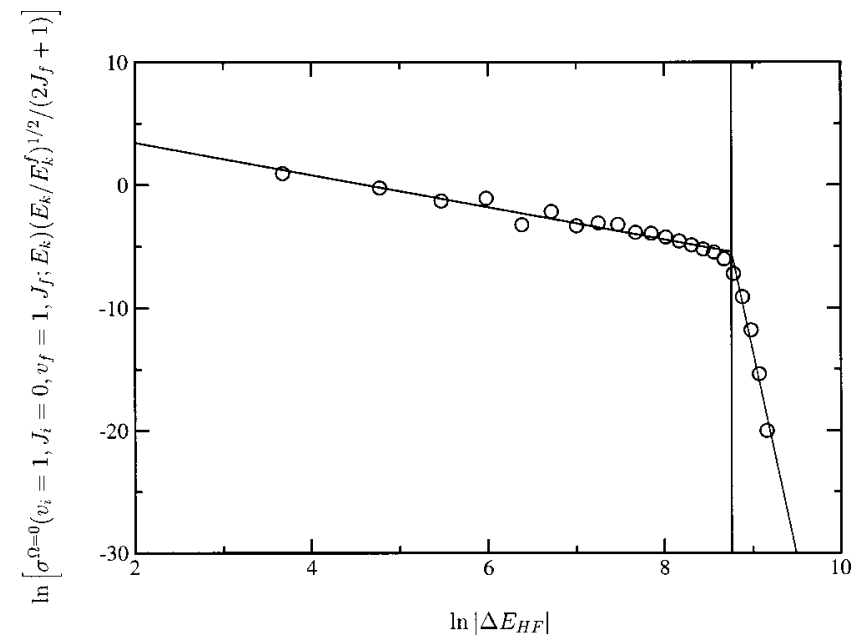

FIG. 6. $\ln \left[\sigma^{\Omega=0}\left(v_{i}=1, J_{i}=0, v_{f}=1, J_{f} ; E_{k}\right)\left(E_{k} / E_{k}^{f}\right)^{1 / 2} /\left(2 J_{f}+1\right)\right]$ vs $\ln \left|\Delta E_{\mathrm{HF}}\right|$ for a relative translational energy of $12878 \mathrm{~cm}^{-1}$ with respect to the $\operatorname{HF}(v=1, J=0)$ state energy. Circles represent final rotational angular momentum states and the vertical line represents the boundary between classically allowed and classically forbidden transitions. It is estimated from the intersection of the two power-gap law data fits. 
sitions with $J_{i}=0$ and $v=1$. It is clear that a single set of parameters could not fit the data well over the entire range.

It should be emphasized that not all energetically open channels are classically allowed. ${ }^{107}$ Some transitions are classically inaccessible due to momentum conservation constraints and from limitations placed by the topology of the surface on angular momentum change. Classically there is a maximum allowable value for the torque-arm (effective impact parameter) in the impulsive limit. At sufficiently high relative velocities, angular momentum conservation is more restrictive than energy conservation, hence a channel can be "open" as far as energy is concerned, and yet be classically forbidden. ${ }^{109,121}$

The angle dependent classical turning point at a relative translation energy of $12878 \mathrm{~cm}^{-1}$ for the HF-Ar intermolecular potential was fitted to an ellipse, as described above. The center of the ellipse was found to be displaced $0.06 \AA$ from the center-of-mass toward the hydrogen and the semimajor and semiminor axes were 1.092 and $1.034 \AA$, respectively. For the ellipsoid model, the maximum classical limit of the angular momentum transfer is, ${ }^{110}$

$$
(\Delta \mathrm{J})_{\max }=\sqrt{2 \mu}\left(\sqrt{E}+\sqrt{E^{\prime}}\right)(A-B)
$$

where $E$ and $E^{\prime}$ are the initial and final translational kinetic energies, $A$ and $B$ are the semimajor and semiminor axes, and $\mu$ is the reduced mass. Using the ellipsoid model for HF-Ar at this energy, the maximum classically allowed change in angular momentum is $(\Delta J)_{\max }=16$, which corresponds to $J_{f}=16$ in Fig. 6.

Agrawal et al., have shown that the intersection of the regressions in Fig. 6 provides an estimate of the boundary between the classically allowed and classically forbidden regions. ${ }^{110}$ The parameters for the power-gap law taken from these regions are $\gamma=1.311, \ln a=6.065$, and $\gamma=33.79, \ln a$ $=290.6$, respectively. This yields an intersection at $(8.762$, -5.422 ) and a classical limit of $6385 \mathrm{~cm}^{-1}$ above the internal energy of the $v=1, J_{i}=0$ state. This agrees well with the ellipsoid model since it also predicts $J_{f}=16$ to be the last classically allowed rotational level. The classically forbidden region extends $6492 \mathrm{~cm}^{-1}$ past the classical limit and includes 8 channels that are open with respect to energy, but closed with respect to angular momentum. The cross sections decrease rapidly with increasing $\left|\Delta E_{\mathrm{HF}}\right|$ in the classically forbidden region.

\section{Vibrational energy transfer}

In order to determine vibrational energy transfer rate constants, collisional cross sections were calculated between the first five vibrational states of hydrogen fluoride using the $V^{2}$ potential. Cross sections $\sigma\left(v_{i}, J_{i}, v_{f}, J_{f} ; E_{k}\right)$ were calculated for all of the open rotational states of each vibrational level. Examples of these cross sections for two relative translational energies are shown in Fig. 7, where a line indicates the locus of V-R state changes that occur without an associated change in internal energy or relative translational energy $\left(\left|\Delta E_{\mathrm{HF}}\right|=\left|\Delta E_{k}\right|=0\right)$. Transitions close to this line exhibit primarily V-R energy transfer where the change in vibrational energy is accompanied by a change in rotational en-
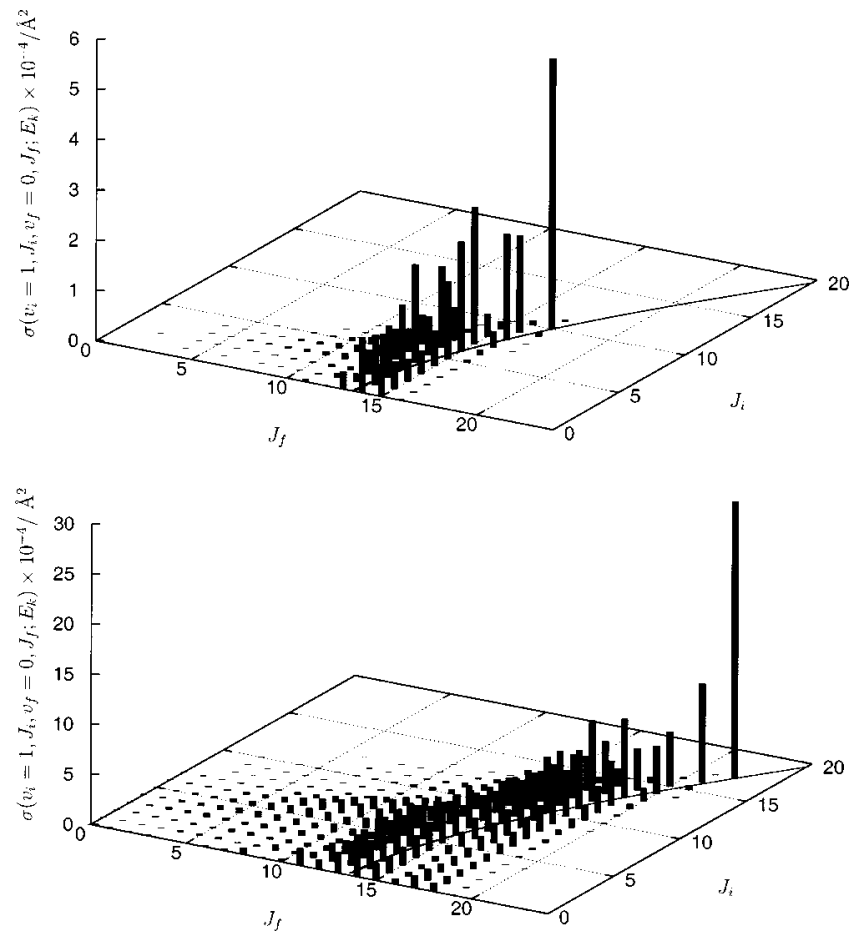

FIG. 7. State-to-state vibrational deactivation cross sections of $\operatorname{HF}(v=1)$ by Ar. The cross sections $\sigma\left(v_{i}=1, J_{i}, v_{f}=0, J_{f} ; E_{k}\right)$ are functions of the relative translational energy $E_{k}$ and depend parametrically on the initial rotational angular momentum quantum number $J_{i}$ and on the final quantum number $J_{f}$. The top graph is for a relative translational energy of 2651.52 $\mathrm{cm}^{-1}$ and the bottom is for $6469.70 \mathrm{~cm}^{-1}$. A line in the $x-y$ plane of both graphs is the locus of V-R state changes that occur with $\left|\Delta E_{\mathrm{HF}}\right|=0$.

ergy of nearly identical magnitude, but of opposite sign; there is virtually no change in translational energy. The graphs show that V-R energy transfer is strongly favored for this system, in agreement with previous findings. ${ }^{18,19}$ For activation, for deactivation, and for transitions where the vibrational state changes by more than one quantum number V-R energy transfer was found to be favored.

To obtain vibrational energy transfer rate constants, cross sections from an initial rotational state $J_{i}$ were summed over the set of all open final states [Eq. (21)]. Calculations were performed for a range of relative translational energies $E_{k}$ resulting in a cross section that depends on the energy and the initial rotational state. An example of these cross sections is shown in Fig. 8 for $J_{i}=0$. For lower energies the cross section exhibits structure that has been associated with resonances. ${ }^{127-133}$ These resonances invert the slope of the cross section as a function of kinetic energy and some individual resonances are evident as spikes on plots of this function. Qualitatively these resonances are due to quasi-bound states as the Ar orbits the HF in the region of the attractive well. However, a full characterization requires analysis ${ }^{127}$ of the $\mathbf{S}$ matrix, which has not been attempted here.

The cross sections $\sigma\left(v_{i}, J_{i}, v_{f} ; E_{k}\right)$ for $\operatorname{Ar}+\mathrm{HF}(v=2$ and $4 ; J_{i}=4,6,8$, and 10) were calculated by Thompson ${ }^{19}$ for a relative translational energy of $5251 \mathrm{~cm}^{-1}$. From his data, Thompson concluded that a relatively small increase in rotation causes a dramatic increase in the cross section for both upward and downward transitions. In a later publication 


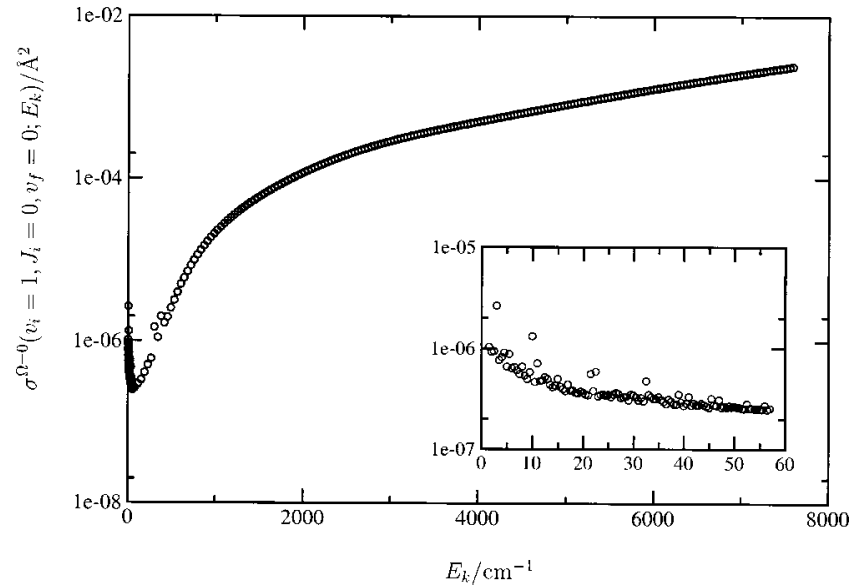

FIG. 8. The cross section $\sigma^{\Omega=0}\left(v_{i}=1, J_{i}=10, v_{f}=0 ; E_{k}\right)$ as a function of the relative translational energy for the vibrational deactivation of $\operatorname{HF}(v=1)$ by Ar. Cross sections are summed over all final $J_{f}$ states. The graphical inset shows the low-temperature region of the plot expanded.

Thompson showed that the trend was not well-behaved, but observed that the cross sections $\sigma\left(v_{i}, J_{i}, v_{f} ; E_{k}\right)$ are strongly dependent on the initial rotational states $J_{i} .{ }^{18}$ The present results are in qualitative agreement with Thompson. The magnitudes of the cross sections are significantly different for varying $J_{i}$ at constant relative translational energy, as shown in Fig. 9 for energies in the neighborhood of that investigated by Thompson. The ordering of the cross sections often changes as a function of relative translational energy.

The predicted vibrational deactivation rate constants for $\operatorname{HF}(v=1)$ as a function of temperature are shown in Fig. 10 for all four potentials. From the figure it is apparent that all of the rate constants are very similar. This is in spite of the different modifications made to the repulsive walls of the potentials and indicates that the calculated results are not very sensitive to the kind of PES enhancing techniques employed here. The original unmodified MP2 ab initio surface performs well and predicts the lowest cross sections. The

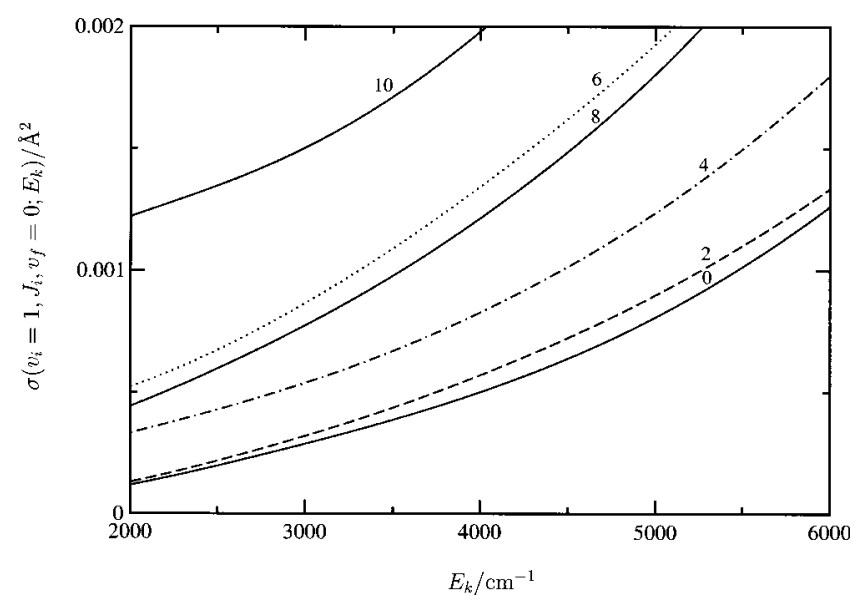

FIG. 9. The cross sections $\sigma^{\Omega=0}\left(v_{i}, J_{i}, v_{f} ; E_{k}\right)$ as a function of relative translational energy for the vibrational deactivation of $\operatorname{HF}(v=1)$ by $\operatorname{Ar}$. Cross sections are summed over all final $J_{f}$ states. Cross sections for $J_{i}$ $>10$ and odd cross sections have been excluded to enhance graphical clarity.

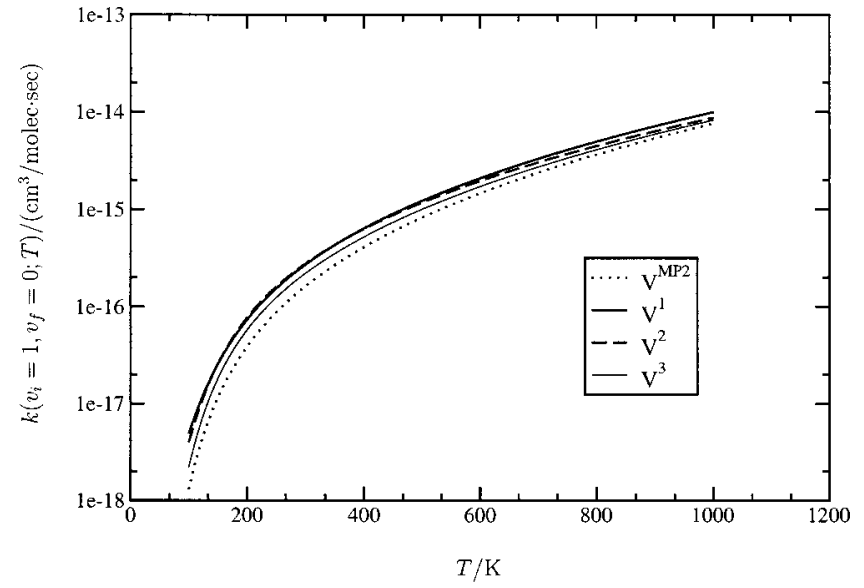

FIG. 10. A comparison of the vibrational deactivation rate constants for all four potentials.

predicted vibrational deactivation rate constant for $\operatorname{HF}(v$ $=1)$ as a function of temperature is shown in Fig. 11, where the theoretical rate constant obtained with the $V^{2}$ potential is presented along with the available experimental data.

As discussed previously, experimental measurement of $\mathrm{HF}(v)$ deactivation by Ar is very difficult at low temperature. Quantitative experimental values only extend down to $800 \mathrm{~K}$ and there are large differences in reports from different groups. The reported experiments at 294 and $350 \mathrm{~K}$ were only able to provide upper limits. In the present work, it was possible to evaluate theoretical rate constants from below $100 \mathrm{~K}$ to as high as $1500 \mathrm{~K}$. Our calculated results are in good agreement with the available experiments, given the spread of the measurements.

At low temperature, the cross-section resonances cause the rate constant to pass through a maximum located around

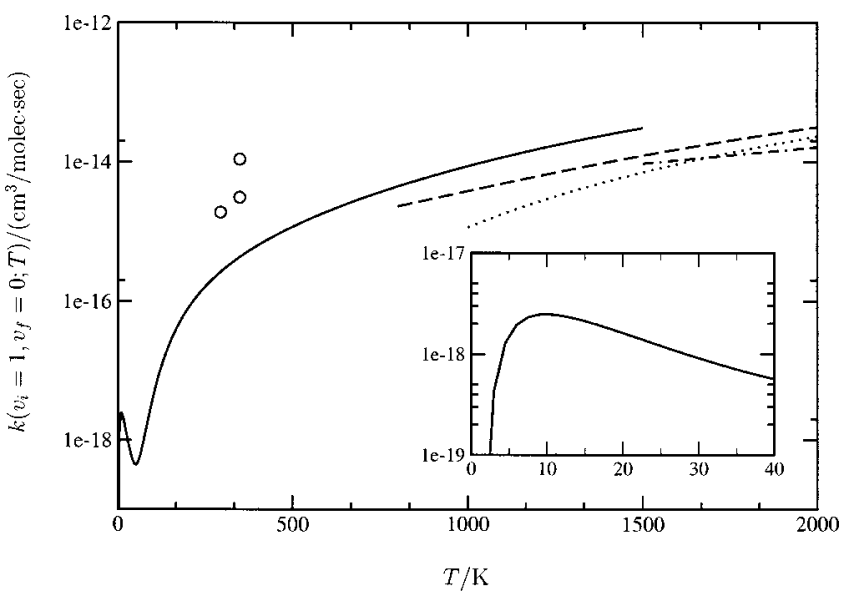

FIG. 11. The vibrational deactivation rate constant for $\operatorname{HF}(v=1)$ collisions with $\mathrm{Ar}$ as a function of temperature. Theoretical data is represented by - the experimental data of Blair et al. (Ref. 15) is represented by - - , the experimental data of Vasil'ev et al. ${ }^{17}$ is represented by $-\cdot-\cdot-\cdot-$, and the experimental data of Bott and Cohen (Ref. 10) is represented by . . . The experimental rate constant upper limits of Hancock and Green (Refs. 11,16), Hinchen (Ref. 14), Airey et al. (Ref. 9), and Fried et al. (Ref. 13) at lower temperature are represented by $\bigcirc$. Hinchen reported the same value for the upper limit at $295 \mathrm{~K}$ as did Hancock and Green, and, therefore, there is only one circle for both. The graphical inset expands the low-temperature region. 
9.4 K, as shown in an expanded scale (see the graphical inset in Fig. 11). The calculated rate constants are less accurate at temperatures below $100 \mathrm{~K}$ due to limitations of the coupledstates approximation and the influence of the resonances. However, the description of the $v=1$ to $v=0$ transition in the 0 to $100 \mathrm{~K}$ temperature regime is in good qualitative agreement with close-coupled calculations that we performed.

\section{CONCLUSIONS}

Theoretical vibrational energy transfer studies require an accurate description of the intermolecular potential energy dependence on the diatomic bond length. The inversion of experimental data is often technically challenging and requires spectroscopic data or collisional energy transfer data that may not exist or may only describe part of the necessary potential surface. The production of theoretical ab initio surfaces is technically and computationally demanding because highly correlated quantum chemistry methods must be employed with large basis sets for many nuclear configurations. Spectroscopically accurate surfaces still only exist for a few systems. The approach taken here was to combine a computationally modest $a b$ initio method with a simple yet effective inclusion of experimental data. The results show that this method is capable of reproducing experimental observations, while at the same time producing a wealth of new detailed information.

All three modified potential energy surfaces perform better than the original MP2 surface for the calculations presented here. Of the three modified surfaces, none is clearly superior for all applications. The $V^{2}$ potential more closely reproduces the experimental binding energies and rotational activation cross sections. However, this potential underestimates the depth of the global minimum at $\theta=0^{\circ}$ (by $\sim 22$ $\mathrm{cm}^{-1}$ ) and overestimates the depth of the local minimum at $\theta=180^{\circ}$ (by $\sim 10 \mathrm{~cm}^{-1}$, see Table I). The $V^{1}$ and $V^{3}$ potentials reproduce the stationary points more accurately and otherwise are similar to each other, with only modest differences in the calculated properties.

Accurate determination of the rotational energy transfer cross sections $\sigma\left(v_{i}, J_{i}, v_{f}, J_{f}, E_{k}\right)$ and $\sigma\left(v_{i}, J_{i}, v_{f}, E_{k}\right)$ required inclusion of an angle-dependent augmentation of the PES. All of the augmented potentials were in good agreement with spectroscopic measurements and with experimental scattering cross sections. The rate constants show many characteristics which are consistent over the temperatures studied: Higher $J$ levels have lower probability of rotational deactivation, downward transitions are favored over upward ones for comparable $\left|\Delta E_{\mathrm{HF}}\right|$, and the rate constants decrease as $\left|\Delta E_{\mathrm{HF}}\right|$ increases. However, the calculated rotational cross sections show complicated behavior and even the relative magnitudes of the state-to-state rate constants depend on translational energy.

The magnitudes of the state-to-state rate constants are subject to both energy conservation and momentum conservation, and they may be limited by either one. The constraints placed on the rotational cross section by momentum affect the rotational energy transfer rate constants as well as the vibrational energy transfer rate constants for the energy range studied. The constraints have the effect of lowering the cross sections and therefore the rate constants. They also complicate the use of empirical fitting functions like the power-gap law.

The vibrational energy transfer rate constants showed little sensitivity to the specific augmented potentials, all three of which give rate constants within a factor of two of those for the MP2 surface. The calculations reaffirm that energy transfer for the HF-Ar system is primarily V-R with little change in internal energy $\left|\Delta E_{\mathrm{HF}}\right|$. This is the case for upward or downward vibrational transitions, transitions where the vibrational state changes by more than one quantum number, and for purely rotational transitions as well. For downward vibrational transitions this would result in a nonBoltzmann increase in the population of the higher rotational levels and the opposite trend for the upward transitions. The vibrational rate constants for $\mathrm{HF}$ are in agreement with experimental values and predictions have been made in the low-temperature regime where experimental measurements are lacking.

\section{ACKNOWLEDGMENTS}

The authors thank Jeremy M. Hutson for providing us with the H6 $4,3,2)$ potential in the form of FORTRAN code and Millard H. Alexander for assistance in porting his quantum scattering code HIBRIDON to the Linux operating system. The authors thank Roman Krems and Alexei A. Buchachenko for technical advice and helpful discussion concerning the diatomic-in-molecule potential. Finally the authors thank M. G. Mlynczak for discussions and NASA (Office of Space Sciences) for funding.

${ }^{1}$ M. G. Mlynczak, J. Geophys. Res., [Space Phys.] 96, 17217 (1991).

${ }^{2}$ M. G. Mlynczak and S. Solomon, Geophys. Res. Lett. 18, 37 (1991).

${ }^{3}$ M. G. Mlynczak and S. Solomon, Geophys. Res. Lett. 18, 1201 (1991).

${ }^{4}$ M. G. Mlynczak and S. Solomon, J. Geophys. Res., [Space Phys.] 98, 10517 (1993).

${ }^{5}$ M. G. Mlynczak, D. K. Zhou, and S. Adler-Golden, Geophys. Res. Lett. 25, 647 (1998).

${ }^{6}$ S. Solomon, J. T. Kiehl, B. J. Kerridge, E. E. Remsberg, and J. M. Russell III, J. Geophys. Res., [Space Phys.] 91, 9865 (1986).

${ }^{7}$ J. A. Kaye, Appl. Opt. 28, 4161 (1989).

${ }^{8}$ S. R. Leone, J. Phys. Chem. Ref. Data 11, 953 (1982).

${ }^{9}$ J. R. Airey and S. F. Fried, Chem. Phys. Lett. 8, 23 (1971).

${ }^{10}$ J. F. Bott and N. Cohen, J. Chem. Phys. 55, 3698 (1971).

${ }^{11}$ J. K. Hancock and W. H. Green, J. Chem. Phys. 56, 2474 (1972).

${ }^{12}$ W. H. Green and J. K. Hancock, IEEE J. Quantum Electron. QE-9, 50 (1973).

${ }^{13}$ S. S. Fried, J. Wilson, and R. L. Taylor, IEEE J. Quantum Electron. QE-9, 59 (1973)

${ }^{14}$ J. J. Hinchen, J. Chem. Phys. 59, 233 (1973).

${ }^{15}$ L. S. Blair, W. D. Breshears, and G. L. Schott, J. Chem. Phys. 59, 1582 (1973).

${ }^{16}$ J. K. Hancock and W. H. Green, J. Chem. Phys. 57, 4515 (1972).

${ }^{17}$ G. K. Vasil'ev, E. F. Makarov, V. G. Papin, and V. L. Talrose, Teor. Mat. Fiz. 64, 2046 (1973).

${ }^{18}$ D. L. Thompson, J. Chem. Phys. 76, 5947 (1982).

${ }^{19}$ D. L. Thompson, Chem. Phys. Lett. 84, 397 (1981).

${ }^{20}$ G. C. Berend and R. L. Thommarson, J. Chem. Phys. 58, 3454 (1973).

${ }^{21}$ D. L. Thompson, J. Chem. Phys. 78, 1763 (1983).

${ }^{22}$ M. Y. Ovchinnikova, Chem. Phys. 93, 101 (1985).

${ }^{23}$ R. V. Krems, N. Marković, A. A. Buchanchenko, and S. Nordholm, J. Chem. Phys. 114, 1249 (2001).

${ }^{24}$ T. F. Ewing, J. Detrich, and R. W. Conn, J. Chem. Phys. 69, 4662 (1978). 
${ }^{25}$ A. A. Buchachenko, N. F. Stepanov, B. L. Grigorenko, and A. V. Nemukhin, J. Chem. Phys. 111, 2470 (1999).

${ }^{26}$ B. L. Grigorenko, A. V. Nemukhin, and V. A. Apkarian, J. Chem. Phys. 104, 5510 (1996).

${ }^{27}$ A. A. Buchachenko, N. F. Stepanov, B. L. Grigorenko, and A. V. Nemukhin, J. Chem. Phys. 111, 2470 (1999).

${ }^{28}$ D. J. Kouri, "Rotational excitation II: Approximation methods," in AtomMolecule Collision Theory: A Guide for the Experimentalist, editors R. B. Bernstein (Plenum, New York, 1979), p. 301.

${ }^{29}$ G. Chalasiński and M. Szczęśniak, Chem. Rev. 7, 1723 (1994).

${ }^{30}$ W. J. Meath and M. Koulis, J. Mol. Struct.: THEOCHEM 72, 1 (1991).

${ }^{31}$ R. J. LeRoy, C. Bissonnette, T. H. Wu, A. K. Dham, and W. J. Meath, Faraday Discuss. 97, 81 (1994).

${ }^{32}$ M. Meuwly and J. M. Hutson, J. Chem. Phys. 110, 8338 (1999).

${ }^{33}$ W. R. Rodwell, L. T. Sin Fai Lam, and R. O. Watts, Mol. Phys. 44, 225 (1981).

${ }^{34}$ M. Jeziorska, P. Jankowski, K. Szalewicz, and B. Jeziorski, J. Chem. Phys. 113, 2957 (2000).

${ }^{35}$ R. J. Le Roy and J. M. Hutson, J. Chem. Phys. 86, 837 (1986).

${ }^{36}$ Advances in Chemical Physics: Intermolecular Forces, edited by J. O. Hirschelder (John Wiley \& Sons, New York, 1967), Vol. 12.

${ }^{37}$ R. Ahlrichs, R. Penco, and G. Scoles, Chem. Phys. 19, 119 (1977).

${ }^{38}$ A. D. Esposti and H.-J. Werner, J. Chem. Phys. 93, 3351 (1990).

${ }^{39}$ M. Yang and M. H. Alexander, J. Chem. Phys. 103, 6973 (1995).

${ }^{40}$ M. H. Alexander, J. Chem. Phys. 111, 7426 (1999).

${ }^{41}$ M. H. Alexander, S. Gregurick, P. J. Dagdigian, G. W. Lemire, M. J. McQuaid, and R. C. Sausa, J. Chem. Phys. 101, 4547 (1994).

${ }^{42}$ H.-J. Werner, B. Follmeg, and M. H. Alexander, J. Chem. Phys. 89, 3139 (1988).

${ }^{43}$ R. A. Kendall, G. Chałasiński, J. Klos, R. Bukowshi, M. W. Severson, M. M. Szczęśniak, and S. M. Cybulski, J. Chem. Phys. 108, 3235 (1998).

${ }^{44}$ J. M. Hutson and B. J. Howard, Mol. Phys. 45, 791 (1982).

${ }^{45}$ J. M. Hutson, J. Chem. Phys. 96, 6752 (1992).

${ }^{46}$ W. Kołos, G. Corongiu, and E. Clementi, Int. J. Quantum Chem. 17, 775 (1980).

${ }^{47}$ F.-M. Tao and W. Klemperer, J. Chem. Phys. 101, 1129 (1994).

${ }^{48}$ T. van Mourik and T. H. Dunning, Jr., J. Chem. Phys. 107, 2451 (1997).

${ }^{49}$ V. F. Lotrich, H. L. Williams, K. Szalewicz, B. Jeziorski, R. Moszynski, P. E. S. Wormer, and Ad vav der Avoird, J. Chem. Phys. 103, 6076 (1995).

${ }^{50}$ D. J. Nesbitt, M. S. Child, and D. C. Clary, J. Chem. Phys. 90, 4855 (1989).

${ }^{51}$ C. Douketis, J. M. Hutson, B. J. Orr, and G. Scoles, Mol. Phys. 52, 763 (1984).

${ }^{52}$ A. K. Rappé and E. R. Bernstein, J. Phys. Chem. 104, 6117 (2000).

${ }^{53}$ M. H. Alexander and P. McGuire, J. Chem. Phys. 64, 452 (1976).

${ }^{54}$ C. Douketis, G. Scoles, S. Marchetti, M. Zen, and A. J. Thakkar, J. Chem. Phys. 76, 3057 (1982).

${ }^{55}$ J. Hepburn, G. Scoles, and R. Penco, Chem. Phys. Lett. 36, 451 (1975)

${ }^{56}$ F. B. Brown and D. G. Truhlar, Chem. Phys. Lett. 117, 307 (1985).

${ }^{57}$ K. Higgins, F.-M. Tao, and W. Klemperer, J. Chem. Phys. 109, 3048 (1998).

${ }^{58}$ J. M. Bowman and B. Gazdy, J. Chem. Phys. 94, 816 (1991).

${ }^{59}$ B. Gazdy and J. M. Bowman, J. Chem. Phys. 95, 6309 (1991)

${ }^{60}$ J. M. Bowman and B. Gazdy, Chem. Phys. Lett. 200, 311 (1992).

${ }^{61}$ J. M. Hutson, A. Ernesti, M. M. Law, C. F. Roche, and R. J. Wheatley, J. Chem. Phys. 105, 9130 (1996).

${ }^{62}$ J. M. Hutson, J. Chem. Phys. 91, 4448 (1989).

${ }^{63}$ W. B. Chapman, M. J. Weilda, and D. J. Nesbitt, J. Chem. Phys. 106, 2248 (1997).

${ }^{64}$ C. M. Lovejoy and D. J. Nesbitt, J. Chem. Phys. 91, 2790 (1989).

${ }^{65}$ M. R. Keenan, L. W. Buxton, E. J. Campbell, A. C. Legon, and W. H. Flygare, J. Chem. Phys. 74, 2133 (1981).

${ }^{66}$ T. A. Dixon, C. H. Joyner, F. A. Baiocchi, and W. Klemperer, J. Chem. Phys. 74, 6539 (1981)

${ }^{67}$ G. T. Fraser and A. S. Pine, J. Chem. Phys. 85, 2502 (1986)

${ }^{68}$ J. T. Farrell, Jr., O. Sneh, A. Mcllroy, A. E. W. Knight, and D. J. Nesbitt, J. Chem. Phys. 97, 7967 (1992)

${ }^{69}$ A. D. Buckingham, P. W. Fowler, and J. M. Hutson, Chem. Rev. 88, 963 (1988).

${ }^{70}$ T. H. Dunning, Jr., J. Chem. Phys. 90, 1007 (1989).

${ }^{71}$ D. E. Woon and T. H. Dunning, Jr., J. Chem. Phys. 100, 2975 (1994).

${ }^{72}$ D. E. Woon and T. H. Dunning, Jr., J. Chem. Phys. 98, 1358 (1993).

${ }^{73}$ NWChem Version 3.3.1, as developed and distributed by Pacific Northwest National Laboratory, P.O. Box 999, Richland, Washington 99352
USA, and funded by the U.S. Department of Energy, was used to obtain some of these results.

${ }^{74}$ A. Szabo and N. S. Ostlund, Modern Quantum Chemistry: Introduction to Advanced Electronic Structure Theory (Macmillan, New York, 1989).

${ }^{75}$ Lecture Notes in Chemistry: Potential Energy Surfaces, edited by A. F. Sax (Springer, Berlin, 1999).

${ }^{76}$ J. N. Murrell, S. Carter, S. C. Farantos, P. Huxley, and A. J. C. Varandas, Molecular Potential Energy Functions (John Wiley \& Sons, New York, 1984).

${ }^{77}$ T. Hollebeek, T.-S. Ho, and H. Rabitz, Constructing multidimensional molecular potential energy surfaces from ab initio data, in Annual Review of Physical Chemistry volume 50, edited by H. L. Strauss (Annual Reviews, Palo Alto, 1999), p. 537.

${ }^{78}$ P. Soldán and J. M. Hutson, J. Chem. Phys. 112, 4415 (2000)

${ }^{79}$ T.-S. Ho and H. Rabitz, J. Chem. Phys. 104, 2584 (1996).

${ }^{80}$ T.-S. Ho and H. Rabitz, J. Chem. Phys. 113, 3960 (2000).

${ }^{81}$ H.-J. Werner and B. Follmeg, J. Chem. Phys. 91, 5425 (1989).

${ }^{82}$ A. D. Esposti, A. Berning, and H.-J. Werner, J. Chem. Phys. 103, 2067 (1995)

${ }^{83}$ C.-C. Chuang, K. J. Higgins, H. C. Fu, and W. Klemperer, J. Chem. Phys. 112, $7022(2000)$

${ }^{84}$ C.-C. Chuang and W. Klemperer, J. Chem. Phys. 113, 4116 (2000).

${ }^{85}$ J. W. Cooley, Math. Comput. 15, 363 (1961).

${ }^{86}$ B. W. Shore, J. Chem. Phys. 59, 6450 (1973).

${ }^{87}$ J. M. Alvariño, L. F. González, M. L. Hernández, and E. Martínez, Spectrosc. Lett. 16, 541 (1983).

${ }^{88}$ J. A. Coxon and P. G. Hajigeorgiou, J. Mol. Spectrosc. 142, 254 (1990).

${ }^{89}$ F. Castaño, J. de Juan, and E. Martinez, J. Chem. Educ. 60, 91 (1983).

${ }^{90}$ LEVel 7.0, "A Computer Program for Solving the Radial Schrödinger Equation for Bound and Quasibound Levels," Copyright 1999 by Robert J. Le Roy, Department of Chemistry, University of Waterloo, Waterloo, Ontario, Canada.

${ }^{91}$ J. M. Hutson, BOUND computer code, version 5 (1993), distributed by Collaborative Computational Project No. 6 of the Science and Engineering Research Council (UK).

${ }^{92}$ J. M. Hutson, Comput. Phys. Commun. 84, 1 (1994).

${ }^{93}$ H.-C. Chang, F.-M. Tao, W. Klemperer, C. Healey, and J. M. Hutson, J. Chem. Phys. 99, 9337 (1993).

${ }^{94}$ D. E. Manolopoulos, J. Chem. Phys. 85, 6425 (1986).

${ }^{95}$ M. H. Alexander and D. E. Manolopoulos, J. Chem. Phys. 86, 2044 (1987).

${ }^{96}$ HIBRIDON ${ }^{\mathrm{TM}}$ is a package of programs for the time-independent quantum treatment of inelastic collisions and photodissociation written by $\mathrm{M}$. H. Alexander, D. E. Manolopoulos, H.-J. Werner, and B. Follmeg, with contributions by P. F. Vohralik, D. Lemoine, G. Corey et al.

${ }^{97}$ M. H. Alexander, J. Chem. Phys. 81, 4510 (1984).

${ }^{98}$ N. Balakrishnan, R. C. Forrey, and A. Dalgarno, Astrophys. J. 514, 520 (1999).

${ }^{99}$ D. R. Flower, E. Roueff, and C. J. Zeippen, J. Phys. B: At., Mol. Opt. Phys. 31, 1105 (1998).

${ }^{100}$ R. T. Pack, J. Chem. Phys. 60, 633 (1974).

${ }^{101}$ P. McGuire and D. J. Kouri, J. Chem. Phys. 60, 2488 (1974).

${ }^{102}$ P. McGuire, Chem. Phys. 13, 81 (1976).

${ }^{103}$ A. E. DePristo and M. H. Alexander, Chem. Phys. 19, 181 (1977).

${ }^{104}$ D. J. Kouri, T. G. Heil, and Y. Shimoni, J. Chem. Phys. 65, 1462 (1976).

${ }^{105}$ D. R. Flower and D. J. Kirkpatrick, J. Phys. B 15, 1701 (1982).

${ }^{106}$ A. J. Banks, D. C. Clary, and H.-J. Werner, J. Chem. Phys. 84, 3788 (1986).

${ }^{107}$ S. Bosanac, Phys. Rev. A 22, 2617 (1980).

${ }^{108}$ I. NoorBatcha and N. Sathyamurthy, Chem. Phys. Lett. 79, 264 (1981).

${ }^{109}$ S. Clare, A. J. Marks, and A. J. McCaffery, J. Chem. Phys. 111, 9287 (1999).

${ }^{110}$ P. M. Agrawal, S. Tilwankar, and N. K. Dabkara, J. Chem. Phys. 108, 4854 (1998).

${ }^{111}$ T. A. Brunner, N. Smith, A. W. Karp, and D. Pritchard, J. Chem. Phys. 74, 3324 (1981).

${ }^{112}$ B. J. Whitaker and P. Brechignac, Chem. Phys. Lett. 95, 407 (1983).

${ }^{113}$ A. J. McCaffery, Z. T. Alwahabi, M. A. Osborne, and C. J. Williams, J. Chem. Phys. 98, 4586 (1993).

${ }^{114}$ P. M. Agrawal and N. C. Agrawal, Chem. Phys. Lett. 118, 213 (1985).

${ }^{115}$ J. A. Serri, R. M. Bilotta, and D. E. Pritchard, J. Chem. Phys. 77, 2940 (1982).

${ }^{116}$ M. A. Osborne and A. J. McCaffery, J. Chem. Phys. 101, 5604 (1994).

${ }^{117}$ P. M. Agrawal and L. M. Raff, J. Chem. Phys. 75, 2163 (1981). 
${ }^{118}$ P. M. Agrawal and L. M. Raff, J. Chem. Phys. 74, 3292 (1981).

${ }^{119}$ J. C. Polanyi and K. B. Woodall, J. Chem. Phys. 56, 1563 (1972).

${ }^{120}$ R. Ramaswamy, A. E. Depristo, and H. Rabitz, Chem. Phys. Lett. 61, 495 (1979).

${ }^{121}$ S. Clare, A. J. Marks, and A. J. McCaffery, J. Phys. Chem. 104, 7181 (2000).

${ }^{122}$ P. M. Agrawal, N. C. Agrawal, and V. Garg, J. Chem. Phys. 83, 4444 (1985).

${ }^{123}$ S. L. Dexheimer, M. Durand, T. A. Brunner, and D. E. Pritchard, J. Chem. Phys. 76, 4996 (1982).

${ }^{124}$ A. J. McCaffery and Z. T. Alwahabi, Phys. Rev. A 43, 611 (1991).

${ }^{125}$ N. C. Lang, J. C. Polanyi, and J. Wanner, Chem. Phys. 24, 219 (1977).
${ }^{126}$ Reinhard Schinke, J. Chem. Phys. 75, 5205 (1981).

${ }^{127}$ J. M. Hutson and F. R. McCourt, J. Chem. Phys. 80, 1135 (1984).

${ }^{128}$ S. Green, J. Chem. Phys. 82, 4548 (1985).

${ }^{129}$ A. Palma and S. Green, J. Chem. Phys. 85, 1333 (1986).

${ }^{130}$ J. P. Reid, C. J. S. M. Simpson, and H. M. Quiney, Chem. Phys. Lett. 256, 531 (1996).

${ }^{131}$ J. P. Reid, C. L. S. M. Simpson, and H. M. Quiney, J. Chem. Phys. 107, 9929 (1997)

${ }^{132}$ J. P. Reid, A. J. Thakkar, P. W. Barnes, E. F. Archibong, H. M. Quiney, and C. J. S. M. Simpson, J. Chem. Phys. 107, 2329 (1997).

${ }^{133}$ J. P. Reid, C. J. S. M. Simpson, and H. M. Quiney, J. Chem. Phys. 106, 4931 (1997). 